\title{
Enhancement of superexchange pairing in the periodically driven Hubbard model
}

\author{
J. R. Coulthard, ${ }^{1}$ S. R. Clark, ${ }^{2,3}$ S. Al-Assam, ${ }^{1}$ A. Cavalleri,,${ }^{1,3}$ and D. Jaksch ${ }^{1,4}$ \\ ${ }^{1}$ Clarendon Laboratory, University of Oxford, Parks Road, Oxford OX1 3PU, United Kingdom \\ ${ }^{2}$ Department of Physics, University of Bath, Claverton Down, Bath BA2 7AY, United Kingdom \\ ${ }^{3}$ Max Planck Institute for the Structure and Dynamics of Matter, University of Hamburg CFEL, Hamburg, Germany \\ ${ }^{4}$ Centre for Quantum Technologies, National University of Singapore, 3 Science Drive 2, Singapore 117543 \\ (Received 13 September 2016; revised manuscript received 25 May 2017; published 1 August 2017)
}

\begin{abstract}
Recent experiments performed on cuprates and alkali-doped fullerides have demonstrated that key signatures of superconductivity can be induced above the equilibrium critical temperature by optical modulation. These observations in disparate physical systems may indicate a general underlying mechanism. Multiple theories have been proposed, but these either consider specific features, such as competing instabilities, or focus on conventional BCS-type superconductivity. Here we show that periodic driving can enhance electron pairing in strongly correlated systems. Focusing on the strongly repulsive limit of the doped Hubbard model, we investigate in-gap, spatially inhomogeneous, on-site modulations. We demonstrate that such modulations substantially reduce electronic hopping, while simultaneously sustaining superexchange interactions and pair hopping via drivinginduced virtual charge excitations. We calculate real-time dynamics for the one-dimensional case, starting from zero- and finite-temperature initial states, and we show that enhanced singlet-pair correlations emerge quickly and robustly in the out-of-equilibrium many-body state. Our results reveal a fundamental pairing mechanism that might underpin optically induced superconductivity in some strongly correlated quantum materials.
\end{abstract}

DOI: 10.1103/PhysRevB.96.085104

\section{INTRODUCTION}

Controlling the structural and electronic properties of a solid by resonantly driving a single low-energy degree of freedom is emerging as a transformative tool in materials science [1]. Such excitations often play a decisive role in stabilizing various broken-symmetry states, and driving them opens up the possibility to switch between phases. This not only includes the melting of equilibrium long-ranged order, such as charge-density waves [2-6], magnetic order [7-9], and orbital order $[9,10]$, but even more remarkably, inducing order, such as superconductivity, out of equilibrium [11,12].

To date, light-induced superconductivity has been observed in several cuprates [13-15] and an alkali-doped fullerene [11] all with quite distinct physics. This raises the question of how ubiquitous such effects are, and what mechanism(s) might underpin their appearance. So far, theoretical exploration has concentrated on a minimal Fröhlich-type model of phononmediated superconductivity $[16,17]$ subjected to a drivinginduced quench of the electronic hopping amplitude. This was envisaged as occurring from a modified electronic structure due to nonlinear phonon coupling $[16,18]$, or from polaronic suppression due to phonon squeezing [17]. In either case, this results in an increase in the density of states at the Fermi level, giving a corresponding increase in the superconducting coupling constant. Despite the slow collective dynamics and elevated electron-phonon scattering, fast enhancements of the superconducting order parameter were predicted.

In this work, we propose a qualitatively different mechanism for driving-enhanced superconductivity in strongly correlated lattice systems. We show that the modulation of site energies in a bipartite lattice with a frequency $\Omega$ inside the charge-transfer gap $U$ slows down electron hopping $t \rightarrow \tilde{t}<t$ without reducing the superexchange interactions $J$ or pair hopping $\alpha J$. This is because the absorption and reemission of quanta from the driving field creates virtual charge-density excitations, leading to additional exchange interactions. Driving-induced contributions, therefore, break the usual relation $J \propto t^{2} / U$, and compensate for the suppressed hopping contribution. As a result, the normally subordinate $J$ can induce a strong pairing effect and enhance long-range pair correlations, even in one spatial dimension. We find that on the moderate time scales assessed, the resulting nonequilibrium states are not significantly heated by the driving field. Instead, driving can substantially reduce the effective temperature of an initial thermal state on experimentally relevant time scales, akin to many-body adiabatic cooling.

Specifically, we investigate the Hubbard model with a bare hopping rate $t$ and a large on-site interaction $U \gg t$, subjected to periodic driving of frequency $\Omega$ that modulates the on-site energy in time $\tau$ with a spatially alternating pattern. We numerically confirm that this leads to dynamical enhancement of pairing for a one-dimensional system with realistic finite-frequency driving $t<\Omega<U$, ramped up on adiabatic and nonadiabatic time scales, and at zero and finite initial temperatures. We also analytically study the system in Floquet theory [19-25] and derive an effective static model to provide qualitative insights into the nonequilibrium dynamics at low temperatures. Note that, in contrast to many studies using Floquet theory, $\Omega$ is not the largest frequency in our setup.

While motivated by experiments, our aim here is to explore a fundamental principle from a minimal, periodically driven, strongly correlated model, as opposed to material-specific $a b$ initio calculations. Nonetheless, organic superconductors (such as charge-transfer salts) under $\mathrm{THz}$ driving are likely candidate systems in which the mechanism proposed here may be observed. In these materials, $\mathrm{THz}$ pulses can resonantly excite an infrared-active local molecular vibration often located in the in-gap regime [26,27]. The ensuing "sloshing" motion of the molecule is sufficiently large in amplitude and heavy that its leading-order effect is to couple to the electronic states via a time-periodic modulation of the on-site energy. 

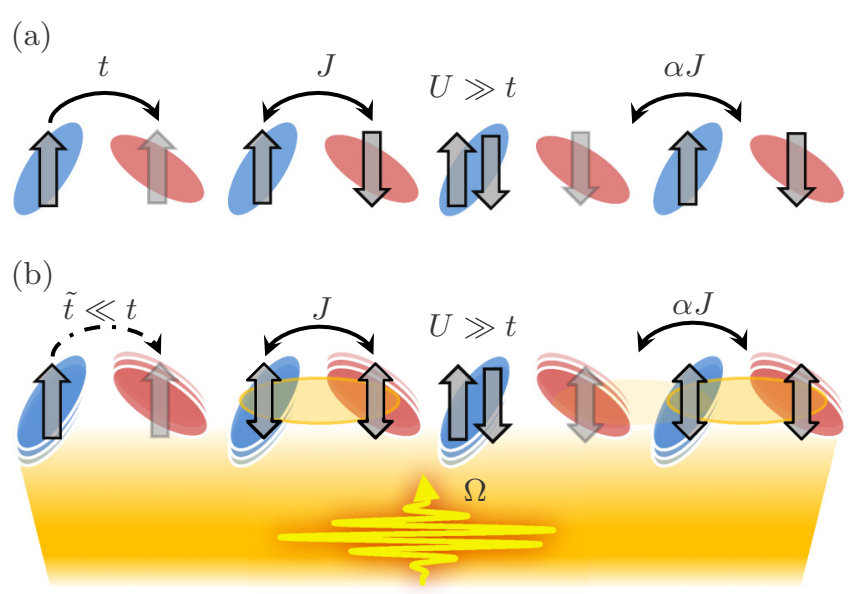

FIG. 1. (a) A bipartite chain with electron hopping $t$, exchange interaction $J$, and on-site correlation $U \gg t$. Singlet pairs move through the lattice with effective hopping rate $\alpha J$. (b) Exciting onsite vibrational modes with $t<\Omega<U$ greatly suppresses hopping $\tilde{t} \ll t$, but leaves superexchange interaction $J$ and pair hopping $\alpha J$ approximately unchanged, resulting in an enhancement of nearestneighbor singlet pairing.

These massive vibrations can therefore be considered classical oscillators for which backaction from the electronic system can be safely ignored. While the laser pulse itself excites coherently and uniformly across the system, the material is assumed to possess a two-molecule unit cell, ${ }^{1}$ as in Fig. 1, so that the modulation induced on the two interlocking sublattices $a$ and $b$ differs in amplitude and/or phase. Such a unit cell might be composed of different molecules or identical molecules with differing orientations due to the stacking morphology [28]. Similar physics can be cleanly realized in optical lattices filled with ultracold fermionic atoms by "shaking" the lattice [29-33], with very recent work going in this direction [34], or by exploiting Raman transitions between internal atomic states [35]. Our results provide a mechanism by which superexchange physics may be better exposed in these systems.

This paper is organized as follows. In Sec. II we introduce the driven Hubbard model, describe the Floquet basis, and work out the quasienergies for a small system via exact numerical diagonalization. We then use time-dependent density matrix renormalization group (DMRG) methods in Sec. III to study the real-time dynamics of the driven Hubbard model in one spatial dimension. In Sec. IV we derive an effective static model whose ground and thermal states are used to approximate the nonequilibrium states of the driven Hubbard model. Finally, we conclude in Sec. V.

\section{THE DRIVEN HUBBARD MODEL}

The focus of this work is the driven Hubbard model Hamiltonian (taking $\hbar=1$ )

$$
\hat{H}(\tau)=\hat{H}_{\text {hub }}+\hat{H}_{\text {drive }}(\tau),
$$

\footnotetext{
${ }^{1}$ For the main effects described here, a two-site periodicity is not essential, and it may be realized with higher spatial periodicities.
}

where $\hat{H}_{\text {hub }}=\hat{H}_{\text {hop }}+\hat{H}_{\text {int }}-\mu \sum_{j} \hat{n}_{j}$, and contributions are given by

$$
\begin{gathered}
\hat{H}_{\text {hop }}=-t \sum_{\langle i j\rangle \sigma}\left(\hat{c}_{i, \sigma}^{\dagger} \hat{c}_{j, \sigma}+\text { H.c. }\right), \\
\hat{H}_{\text {int }}=U \sum_{j} \hat{n}_{j, \uparrow} \hat{n}_{j, \downarrow}, \\
\hat{H}_{\text {drive }}(\tau)=\frac{V_{a}}{2} \sin (\Omega \tau-\Delta \phi) \sum_{j \in a} \hat{n}_{j} \\
+\frac{V_{b}}{2} \sin (\Omega \tau+\Delta \phi) \sum_{j \in b} \hat{n}_{j} .
\end{gathered}
$$

Here $\hat{c}_{i, \sigma}$ with $\sigma=\uparrow, \downarrow$ is the fermionic annihilation operator for a spin- $\sigma$ electron on site $j, \hat{n}_{j, \sigma}=\hat{c}_{j, \sigma}^{\dagger} \hat{c}_{j, \sigma}, \hat{n}_{j}=\hat{n}_{j, \uparrow}+$ $\hat{n}_{j, \downarrow}$, and $\langle i j\rangle$ denotes nearest-neighbor sites on a bipartite lattice composed of $a$ and $b$ sublattices. We denote the lattice filling by $\bar{n}=\sum_{j}\left\langle\hat{n}_{j}\right\rangle / L$, where $L$ is the number of lattice sites. The hopping amplitude is $t, U$ is the on-site Coulomb repulsion, and $\mu$ is the chemical potential. The driving $\hat{H}_{\text {drive }}(\tau)$ describes a time $\tau$ periodic single-particle Hamiltonian with driving frequency $\Omega$, corresponding sublattice driving amplitudes $V_{a(b)}$, and a phase difference of $2 \Delta \phi$. For simplicity, we assume $V_{a}=V_{b}=V$ for the driving amplitude, which may be a function of time $V(\tau)$, and a constant phase $\Delta \phi=\pi / 2$ throughout the paper. However, the qualitative features of our results are expected more generally (see Appendix A). We next use a Floquet analysis to start investigating the dynamics induced by $\hat{H}(\tau)$.

\section{A. Floquet analysis}

Floquet theory $[19,22]$ is based on the time ana$\log$ of Bloch's theorem and is applicable here since $\hat{H}(\tau+T)=\hat{H}(\tau)$ with $T=2 \pi / \Omega$. It states that a complete set of solutions to the time-dependent Schrödinger equation $\left[\hat{H}(\tau)-i \partial_{\tau}\right]|\Psi(\tau)\rangle=0$ can then be written as $\left|\psi_{\eta}(\tau)\right\rangle=\exp \left(-i \epsilon_{\eta} \tau\right)\left|\phi_{\eta}(\tau)\right\rangle$. The $T$-periodic Floquet states $\left|\phi_{\eta}(\tau+T)\right\rangle=\left|\phi_{\eta}(\tau)\right\rangle$ are solutions to the eigenvalue equation

$$
\left[\hat{H}(\tau)-i \partial_{\tau}\right]\left|\phi_{\eta}(\tau)\right\rangle=\epsilon_{\eta}\left|\phi_{\eta}(\tau)\right\rangle,
$$

with associated real quasienergies $\epsilon_{\eta}$ that are defined up to integer multiples of $\Omega$. Periodicity means that the quasienergy spectrum possesses a zonelike structure where physically distinct eigenstates lie within a quasienergy range $E-\frac{1}{2} \Omega<$ $\epsilon_{\eta} \leqslant E+\frac{1}{2} \Omega$, where the choice of $E$ is arbitrary but often taken as $E=0$.

The Hermitian Floquet Hamiltonian $\hat{H}_{\mathrm{F}}=\hat{H}(\tau)-i \partial_{\tau}$ acts on an extended Hilbert space $\mathcal{H} \otimes \mathcal{T}$, which augments the original Hilbert space $\mathcal{H}$ by the space $\mathcal{T}$ of square-integrable $T$-periodic functions in time. This extended Hilbert space, whose elements are denoted as $|\chi\rangle\rangle$, is endowed with a suitable scalar product by time-averaging over a period $T$ as

$$
\langle\langle\chi \mid \xi\rangle\rangle=\frac{1}{T} \int_{0}^{T}\langle\chi(\tau) \mid \xi(\tau)\rangle d \tau,
$$

where $|\chi(\tau)\rangle$ and $|\xi(\tau)\rangle$ are any $T$-periodic states in $\mathcal{H}$, and $\langle\chi(\tau) \mid \xi(\tau)\rangle$ is the conventional scalar product for $\mathcal{H}$. 
We take the Fock basis $\left|\left\{n_{j, \sigma}\right\}\right\rangle$ of the lattice system, where $n_{j, \sigma}$ spin- $\sigma$ electrons occupy site $j$, and we construct an orthonormal Floquet-Fock basis of $\mathcal{H} \otimes \mathcal{T}$ as [25]

$$
\left.\left|\left\{n_{j, \sigma}\right\}, m\right\rangle\right\rangle=\left|\left\{n_{j, \sigma}\right\}\right\rangle e^{i m \Omega \tau+i \frac{V}{2 \Omega} \sin (\Omega \tau)\left(\sum_{j \in a} n_{j}-\sum_{j \in b} n_{j}\right)} .
$$

These basis states include phases for the $m$ th Fourier component and those associated with transforming into the frame rotating with $\hat{H}_{\text {drive }}(\tau)$.

The matrix elements of the Floquet Hamiltonian $\hat{H}_{\mathrm{F}}$ in this basis are

$$
\begin{aligned}
& \left\langle\left\langle\left\{n_{j, \sigma}^{\prime}\right\}, m^{\prime}\left|\hat{H}_{\mathrm{F}}\right|\left\{n_{j, \sigma}\right\}, m\right\rangle\right\rangle \\
& =\zeta_{m^{\prime}-m}\left\langle\left\{n_{j, \sigma}^{\prime}\right\}\left|\hat{H}_{\mathrm{hop}}\right|\left\{n_{j, \sigma}\right\}\right\rangle \\
& \quad+\delta_{m, m^{\prime}}\left[\left\langle\left\{n_{j, \sigma}^{\prime}\right\}\left|\hat{H}_{\mathrm{int}}\right|\left\{n_{j, \sigma}\right\}\right\rangle+m \Omega\right] .
\end{aligned}
$$

The couplings $\zeta_{m^{\prime}-m}$ are given by (also see Appendix A)

$$
\zeta_{m^{\prime}-m}=s^{m^{\prime}-m} \mathcal{J}_{m^{\prime}-m}(v),
$$

where $\mathcal{J}_{n}$ is the $n$ th-order Bessel function of the first kind, and $v=V / \Omega$. They depend on the difference in the Fourier components $m^{\prime}-m$, and also on the driving parameter $v$ as well as the change in sublattice $a$ occupation $s=\sum_{j \in a}\left(n_{j}^{\prime}-\right.$ $\left.n_{j}\right)= \pm 1$ for the Fock states being connected.

This matrix representation of $\hat{H}_{\mathrm{F}}$ has a natural block structure with respect to the Fourier index $m$ labeling the Floquet sector replicas of the system. The diagonal blocks are a matrix representation of $\mathcal{J}_{0}(v) \hat{H}_{\text {hop }}+\hat{H}_{\text {int }}$ in the Fock basis, i.e., $\hat{H}_{\text {hub }}$ with a renormalized hopping amplitude $\tilde{t} \equiv$ $t \mathcal{J}_{0}(v)$, and shifted in energy by $m \Omega$. Correspondingly, the off-diagonal blocks coupling different $m$ sectors are a matrix representation of $\zeta_{m^{\prime}-m} \hat{H}_{\text {hop. }}$. In the remainder of this section, we numerically investigate the Floquet Hamiltonian for a small system.

\section{B. Small system}

Using a small, numerically exactly diagonalizable onedimensional lattice, we calculate the quasienergy spectrum $\epsilon_{\eta}$ as a function of $v$ from Eq. (5). We concentrate on the quasienergy states in the $m=0$ Floquet sector that

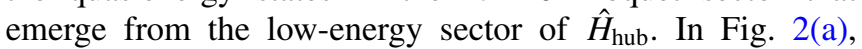
the results for high-frequency driving $\Omega \gg U, t$, where the Floquet sectors are energetically well separated and decouple in a perturbative sense. The width of the spectrum initially shrinks with increasing $v$, indicating a reduction of the driven hopping amplitude $\tilde{t}<t$, consistent with the $\zeta_{0}=$ $\mathcal{J}_{0}(v)$ dependence. When the driving strength reaches $v=$ $v_{0} \approx 2.4$, where $\mathcal{J}_{0}\left(v_{0}\right)=0$, the electron hopping and the superexchange are both fully suppressed. The dynamics of the system are therefore frozen, as signified by the collapse of the spectrum to an $\epsilon_{\eta}=0$ degeneracy at $\nu_{0}$. With further increases of $v$, the hopping amplitude $\tilde{t}$ becomes negative, and the quasienergy spectrum correspondingly broadens. This so-called "dynamical localization" and band-flipping are the well-known single-particle effect that has been demonstrated experimentally, e.g., in optical lattices [20,31,36].

A qualitatively different result occurs for in-gap driving $t<\Omega<U$, as shown in Fig. 2(b). The quasienergy spectrum is again seen to reduce in bandwidth with increasing $v$

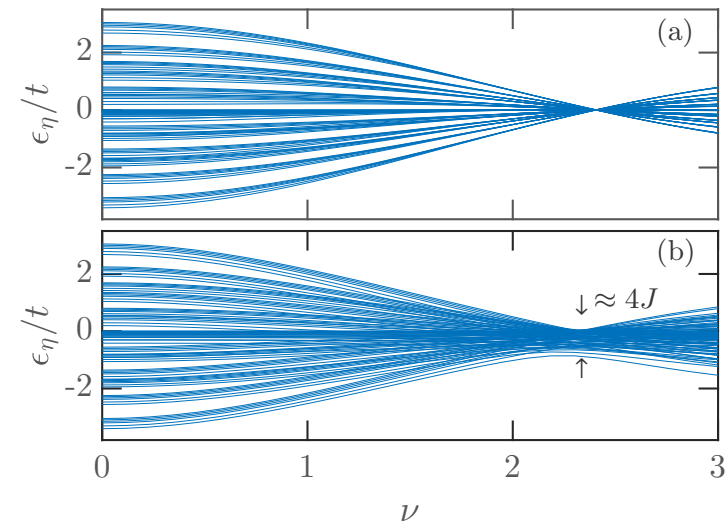

FIG. 2. The singly occupied subspace of the Floquet quasienergy spectrum $\epsilon_{\eta}$, computed exactly for $L=6$ sites, with two up-electrons and two down-electrons. In (a), where $\Omega=100 t$ and $U=20 t$, the quasienergy spectrum collapses to a single point at $v_{0} \approx 2.4$, signaling complete suppression of $\tilde{t}$ relative to $U$. In (b), where $\Omega=6 t$ and $U=$ $20 t$, the spectrum does not collapse to a point, but instead strongly resembles the spectrum of a $t-J$ Hamiltonian with $J>\tilde{t}$.

initially, indicating that $\tilde{t}$ is still being suppressed. However, in contrast to the high-frequency limit the spectrum retains a finite width proportional to $J=4 t^{2} / U$, and it is shifted down by approximately $J$, even when $v \approx v_{0}$. Rather than being frozen out, the dynamics in this driving regime are now being governed by the normally subordinate superexchange energy $J$ that appears to be unsuppressed. This observation motivates the further numerical studies presented in the next section. There we gather evidence that the driven system has an increased susceptibility to pair formation and long-range correlations for driving $v \lesssim v_{0}$. This then leads us to derive an effective static $t$ - $J$ model that describes the singlet-pair dynamics in the driven state with good accuracy even for strong driving, $v>1$.

\section{DRIVING-ENHANCED FERMION PAIRING}

Here we consider a one-dimensional driven Hubbard model, and we study directly its real-time dynamics when slowly ramping up the driving amplitude, first at zero temperature for both infinite and finite systems, and then at finite temperature for a finite system. Our numerics are based on highly accurate time-dependent DMRG methods [37-40] as implemented in the Tensor Network Theory (TNT) Library [41], and they are described in more detail in Appendix C.

\section{A. Zero temperature}

We calculate the real-time dynamics for the translationally invariant infinite system starting from the ground state, using a chemical potential $\mu=-0.2 t$, resulting in an approximately quarter-filled system. The driving amplitude is ramped up with in-gap driving frequency $t<\Omega<U$. We characterize the driving-induced nonequilibrium state $|\psi(\tau)\rangle$ by studying its density-density correlations

$$
N_{i j}(\tau)=\left\langle\hat{n}_{i} \hat{n}_{j}\right\rangle-\left\langle\hat{n}_{i}\right\rangle\left\langle\hat{n}_{j}\right\rangle
$$



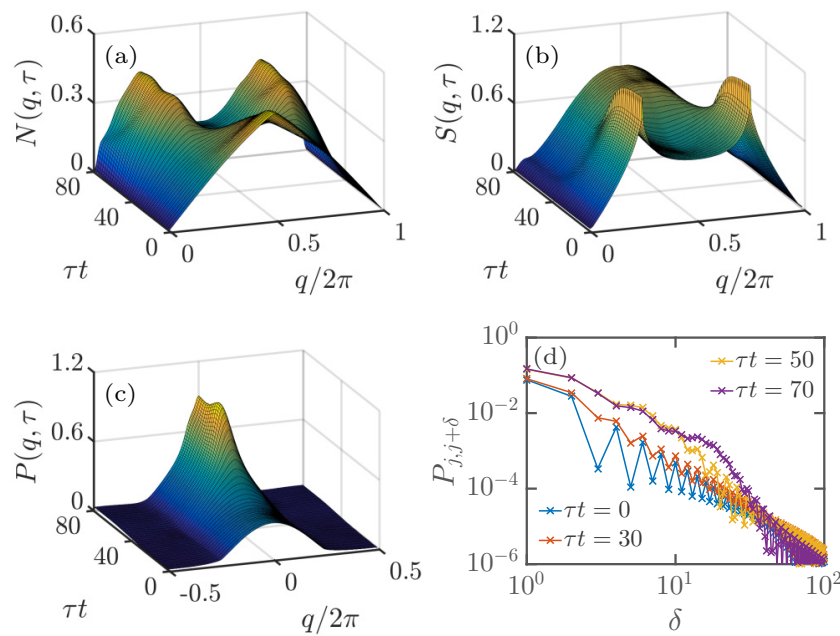

FIG. 3. An infinite Hubbard chain with $U=20 t, \mu=-0.2 t$, and driving frequency $\Omega=6 t$. The driving amplitude is ramped according to $v(\tau)=[v(\infty) / 2]\left\{\tanh \left[\left(\tau-\tau_{0}\right) / \tau_{\mathrm{r}}\right]+1\right\}$ with final driving strength $v(\infty)=2.2$, ramp time $\tau_{\mathrm{r}}=15 / t$, and $\tau_{0}=25 / t$. The plots show (a) the density-density structure factor $N(q, \tau)$, (b) the spin structure factor $S(q, \tau)$, and (c) the singlet pairing structure factor $P(q, \tau)$ as a function of quasimomentum $q$ and time $\tau$. In (a)-(c) we have averaged over the frequency $\Omega$ oscillations, e.g., those that are visible in the line-outs shown in Fig. 4(a). Residual low-frequency oscillations in these quantities are due to the finite ramping time $\tau_{\mathrm{r}}$. In (d) the real-space pairing correlation function $P_{j, j+\delta}$ at various time slices is shown.

the spin-spin correlations

$$
S_{i j}(\tau)=\left\langle\hat{S}_{i}^{z} \hat{S}_{j}^{z}\right\rangle
$$

with $\hat{S}_{i}^{z}=\left(\hat{n}_{i, \uparrow}-\hat{n}_{i, \downarrow}\right) / 2$, and the nearest-neighbor singletparing correlations

$$
P_{i j}(\tau)=\left\langle\hat{b}_{i, i+1}^{\dagger} \hat{b}_{j, j+1}\right\rangle .
$$

Here the operators $\hat{b}_{i j}^{\dagger}\left(\hat{b}_{i j}\right)$, given by

$$
\hat{b}_{i j}^{\dagger}=\frac{1}{\sqrt{2}}\left(\hat{c}_{i, \uparrow}^{\dagger} \hat{c}_{j, \downarrow}^{\dagger}-\hat{c}_{i, \downarrow}^{\dagger} \hat{c}_{j, \uparrow}^{\dagger}\right),
$$

create (annihilate) a singlet electron pair at sites $i$ and $j$, and $\langle\cdot\rangle=\langle\psi(\tau)|\cdot| \psi(\tau)\rangle$. We mostly concern ourselves with the corresponding structure factors,

$$
X(q)=\sum_{k} X_{j, j+k} e^{i q k},
$$

for the translationally invariant infinite system, where $X$ is any of the quantities $N, S$, or $P$, and $q$ is the dimensionless quasimomentum.

We plot the density structure factor $N(q, \tau)$ in Fig. 3(a). The initial kink at $q=4 k_{\mathrm{F}}$, where $k_{\mathrm{F}}=\bar{n} \pi / 2$ is the noninteracting Fermi wave vector, is suppressed in favor of peaks at $q= \pm 2 k_{\mathrm{F}}$ as the driving increases. This signifies a doubling of the wavelength of Friedel oscillations in the system's densitydensity correlations and is indicative of the formation of bound pairs. We interpret the slope of $N(q, \tau)$ as $q \rightarrow 0$ as a dynamical version of the Luttinger parameter $K_{\rho}(\tau)$ [42].
In equilibrium $N(q) \approx K_{\rho} q / \pi[43,44]$, and in Fig. 3(a) it is apparent that the gradient around $q=0$ increases at later times corresponding to an increase in $K_{\rho}(\tau)$. Eventually, $K_{\rho(\tau)}$ exceeds 1 signifying the formation of an attractive Luttinger liquid.

The spin structure factor, shown in Fig. 3(b), begins with sharp peaks at $2 k_{\mathrm{F}}$, indicating that the Hubbard ground state has a tendency toward antiferromagnetic order. At quarterfilling, this order is incommensurate with the lattice period. In the presence of periodic driving, the initial peaks are suppressed and instead a peak at $q=\pi$ forms, consistent with the formation of islands of commensurate antiferromagnetic order. The broadness of this emerging peak shows that the underlying order is not quasi-long-ranged yet. Indeed, its form is similar to $S(q)=\bar{n}(1-\cos q)$ expected for a gas of free nearest-neighbor singlet pairs [45].

We obtain the most direct evidence of pairing via the singlet structure factor, and in particular its uniform $P(q=0, \tau)$ component, which contains contributions from both longrange and short-range correlations. In Fig. 3(c), a broad peak about $q=0$ is seen initially, which under driving eventually increases in magnitude by a factor of more than 3 , and sharpens. This is consistent with $K_{\rho}(\tau)>1$ and suggests that the driving has formed a quasicondensate of singlet pairs in momentum space. To isolate the long-range contribution to $P(q=0, \tau)$, we examine the real-space singlet correlations in Fig. 3(d). These correlations confirm a suppression of $2 k_{\mathrm{F}}$ modulations at short times, followed by a significant enhancement of the pairing correlations. They are found to spread through the system at a rate of approximately $t / 4 \sim J$, consistent with a pair-hopping $\alpha J$ as shown in Fig. 1 [46]. We observe that the enhancement reaches a range of approximately 20-30 sites on the time scales considered.

In Fig. 4 we demonstrate the robustness of the pairing dynamics for different driving parameters. As shown in Fig. 4(a), the magnitude of $P(q=0, \tau)$ oscillates with frequency $\Omega$ about a mean value that is greatly enhanced with increasing driving strength $v$ as long as $v<v_{0}$. The dynamical Luttinger parameter displayed in Fig. 4(b) increases with driving and exceeds unity for sufficiently strong driving. This suggests the onset of attractive interactions within the system. However, for the largest final driving strengths $v(\infty) \approx v_{0}$, where the tunneling is suppressed most strongly, $P(q=0, \tau)$ reaches a maximum height at intermediate times and then reduces. This behavior is indicative of the pair state being unstable to decay toward a resonating valence bond (RVB) type state, as we will discuss in detail in the next section.

The dependence of $P(q=0, \tau)$ on the ramping time $\tau_{\mathrm{r}}$ is shown in Fig. 4(c). Roughly speaking, the ramp is expected to cross over to adiabatic when the time derivative of its amplitude $\dot{v} \approx \nu(\infty) / \tau_{r} \approx J$, i.e., when it is sufficiently smaller than the dominant energy scale of the final Hamiltonian. This is not satisfied for the fastest ramps shown in Fig. 4(c), yet within their ramping profile a moderate enhancement of the pairing is still induced and is sustained for longer times. For the slowest ramps considered, the increases in the enhancement begin to saturate, suggesting they are close to adiabatic for this system.

In Fig. 4(d) we show the final pairing enhancement as a function of driving frequency. A number of resonance dips are seen where no singlet-pairing enhancement is observed. 

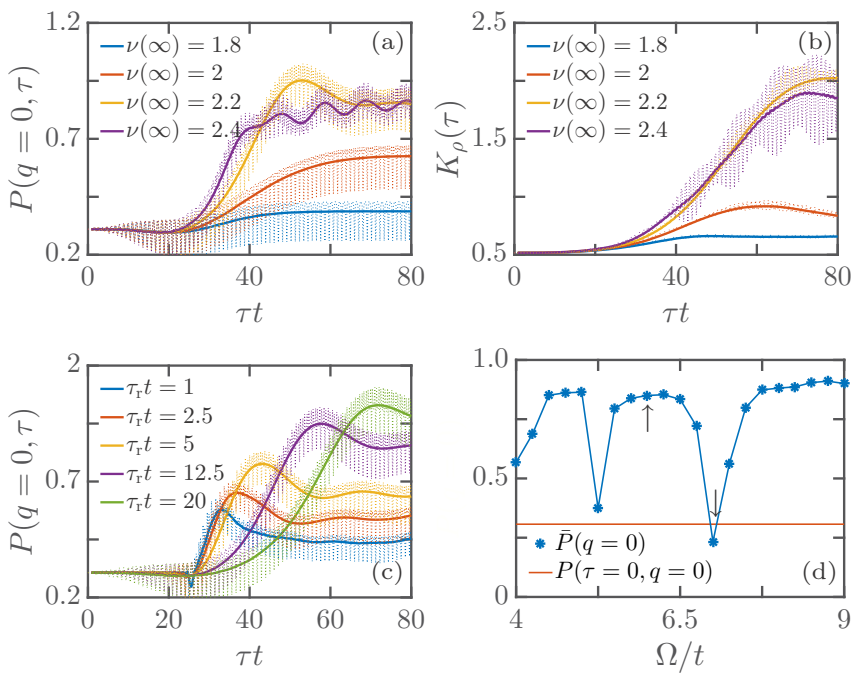

FIG. 4. (a) The height of the singlet structure factor peak $P(q=$ $0, \tau)$ at various final driving strengths. The instantaneous value is indicated by a dotted line, while the moving time average is denoted by a solid line. (b) The dynamical Luttinger parameter $K_{\rho}(\tau)$ extracted from the slope of $N(q, \tau)$ at $q \rightarrow 0$. (c) The peak of the singlet structure factor $P(q=0, \tau)$ for various ramp times. (d) The final height of the singlet structure factor averaged from $\tau=60 / t$ to $80 / t$, $\bar{P}(q=0)$ as a function of the driving frequency $\Omega$. The solid blue line is drawn to guide the eye. Arrows mark frequencies $\Omega=6 t$ and $7 t$. All parameters not explicitly given in the plots are the same as in Fig. 3.

The origin of these is traced back to level crossings in the Floquet quasienergy spectrum as the ramp is traversed, which is discussed in more detail in Appendix B. In short, at these frequencies $m>0$ Floquet replicas of the upper Hubbard band, composed of states containing high-energy doubly occupied configurations, cross the $m=0$ lower Hubbard band, composed of states with predominantly singly occupied configurations. As a result, the driving induces resonant transitions between these states and $m \Omega$ of energy is absorbed, creating double-occupancies and destroying nearestneighbour pairs. However, away from these resonances the substantial enhancement reported for $\Omega=6 t$ is observed over a wide range of frequencies $\Omega<U$.

Given the relatively slow speed of the spread of pairing correlations shown in Fig. 3(a), we expect that a finite system of a few 10's of sites will adequately capture the physics of the driven system, particularly for finite-temperature systems where the range of correlations is suppressed by thermal fluctuations. Indeed, in Appendix $\mathrm{C}$ we directly compare infinite and finite time-dependent DMRG calculations and confirm that there is a negligible difference between the structure factors of the infinite and finite systems.

\section{B. Finite temperature}

The relevance of our observations at zero temperature to real materials hinges on whether this effect survives at finite temperatures. To answer this, we use the finite-temperature extension to time-dependent DMRG $[47,48]$. We then compute the coherent evolution with periodic driving via $\hat{H}(\tau)$ for an initial thermal state of $\hat{H}_{\text {hub }}$ at inverse temperature $\beta_{0}$. For
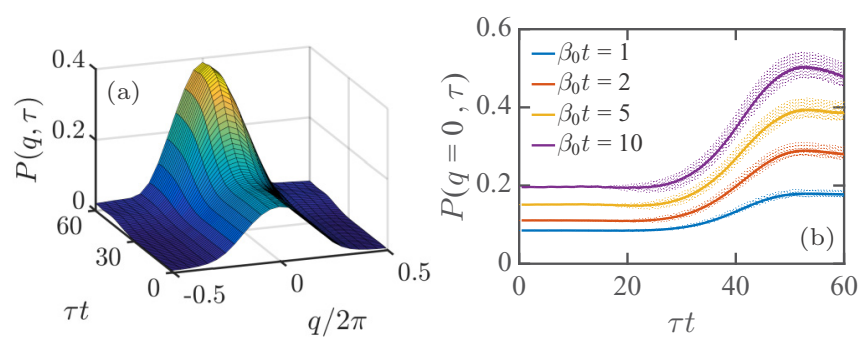

FIG. 5. (a) The singlet structure factor $P(q, \tau)$ with an initial temperature $\beta_{0}=5 / t$. As in Fig. 3, we have averaged over the frequency $\Omega$ oscillations. (b) The height of the peak $P(q=0, \tau)$ for several initial temperatures $\beta_{0}$. The dotted line shows the instantaneous value, while the solid line shows the moving time average. These results were computed for $L=24$ sites. All other parameters not explicitly given in the plots are the same as in Fig. 3.

concreteness, we restrict our considerations here to a finite system of $L$ sites.

Figure 5(a) shows that enhanced pairing persists at finite temperature, albeit with a peak of reduced height and broadened width compared to the zero-temperature case. In Fig. 5(b), we show the singlet structure factor $P(q=$ $0, \tau)$ as a function of time for various initial values of $\beta_{0}$. Perhaps counterintuitively, the driven state consistently exhibits enhanced singlet pairing correlations even when the initial temperature $1 / \beta_{0} \sim t \gg J$ far exceeds the pair binding energy. In the next section, we will introduce an effective time-independent model to qualitatively capture all the physics underlying the numerical results discussed so far. In the context of this effective model, the thermal enhancement can be viewed as a many-body version of adiabatic cooling.

The reason for this is already apparent from Fig. 2(b). As the driving amplitude $v$ approaches $v_{0}$, the bandwidth of the quasienergy spectrum in the $m=0$ Floquet sector is squashed. This is described by changes in the effective model's parameters with $v$, and from Fig. 4(c) we saw that for sufficiently slow ramping, $\dot{v} \ll J$, this change will be adiabatic. Consequently, the driving substantially reduces the energy gaps between the many-body eigenstates of this model while keeping their thermal populations unchanged. Therefore, the driven state remains approximately thermal, but at a significantly lower temperature. A similar effect is used in cold-atom systems, where an adiabatic increase of the lattice depth results in a lowering of the temperature [49]. Indeed it has been shown that even for instantaneous quenches, one can obtain cooling in a wide variety of physical systems [50].

\section{EFFECTIVE $t$ - $J$ MODEL}

The numerical results presented in the previous section indicate that superexchange interaction $J$ and pair hopping $\alpha J$, as in Fig. 1, play a significant role in the driven dynamics when $t<\Omega<U$. Moreover, from our calculations we find [see Fig. 7(b)] that the negligible double occupation in the initial state remains small in the driven state, once $\Omega$ does not coincide with any resonance. This suggests that an effective $t-J$ model may represent an adequate foundation for a Floquet analysis in this driving regime [51]. The $t-J$ model arises from 
$\hat{H}_{\text {hub }}$ by perturbatively projecting double occupancies out via the standard approach [52] to yield

$$
\hat{H}_{\mathrm{t} J \alpha}=\mathbb{P}_{0}\left[\hat{H}_{\text {hop }}+\hat{H}_{\mathrm{ex}}+\hat{H}_{\text {pair }}\right] \mathbb{P}_{0},
$$

with superexchange and pair hopping contributions

$$
\begin{gathered}
\hat{H}_{\mathrm{ex}}=-J \sum_{\langle i j\rangle} \hat{b}_{i j}^{\dagger} \hat{b}_{i j}, \\
\hat{H}_{\mathrm{pair}}=-\alpha J \sum_{\langle i j k\rangle}^{i \neq k}\left(\hat{b}_{i j}^{\dagger} \hat{b}_{j k}+\text { H.c. }\right) .
\end{gathered}
$$

Here, the operator $\mathbb{P}_{0}=\prod_{j=1}^{L}\left(1-\hat{n}_{j, \uparrow} \hat{n}_{j, \downarrow}\right)$ projects onto the subspace of Fock states without any double occupancies. The bracket $\langle i j k\rangle$ denotes sums over nearest-neighbor sites, not double-counting bonds. The two-site superexchange $\hat{H}_{\mathrm{ex}}$ term binds nearest-neighbor singlet pairs together with an energy $J$. Correspondingly, the three-site pair hopping term $\hat{H}_{\text {pair }}$ describes the motion of these pairs without breaking their bond.

In equilibrium, the parameters of the $t-J$ model Hamiltonian $\hat{H}_{t J \alpha}$ relate to the original Hubbard model through $J=4 t^{2} / U$ and $\alpha=1 / 2$. The validity of the $t-J$ model relies on the strongly interacting limit $t \ll U$, and thus mandates $J \ll t$. We now proceed with a Floquet analysis of the driven $t$ - $J$ Hamiltonian defined as

$$
\hat{H}^{\prime}(\tau)=\hat{H}_{t J \alpha}+\mathbb{P}_{0} \hat{H}_{\text {drive }}(\tau) \mathbb{P}_{0},
$$

identical to that described in Sec. II A. Removing $U$ as an explicit energy scale allows us to examine the behavior with $\Omega$ as the largest energy scale of the resulting model, while continually assuming in-gap driving $\Omega<U$. This approach will provide us with a simple, intuitive picture of the physics for in-gap driving. A more detailed description, handling the interplay between finite $U$ and $\Omega$ in perturbation theory, is contained in Appendix E. There, starting from the driven Hubbard model, we explicitly work out the contributions to the exchange interaction from the electron hopping and from driving-induced virtual charge excitations. Those calculations corroborate the picture arising from the $t-J$ model for sufficiently weak driving strength $v$ and $\Omega<U$.

\section{A. Floquet analysis}

By calculating a matrix representation of the Floquet Hamiltonian for the driven $t-J$ model $\hat{H}^{\prime}(\tau)$, using the Floquet basis introduced in Sec. II A, we immediately find that the diagonal blocks are given by the matrix representation of $\mathbb{P}_{0}\left[\mathcal{J}_{0}(v) \hat{H}_{\text {hop }}+\hat{H}_{\text {ex }}+\hat{H}_{\text {pair }}\right] \mathbb{P}_{0}$. Thus, the hopping is still suppressed, while the driving-dependent phase factors in the Floquet basis cancel out for $\hat{H}_{\text {ex }}$ and $\hat{H}_{\text {pair }}$, as they did for $\hat{H}_{\text {int }}$ in the driven Hubbard model, leaving these terms unaffected. Assuming $\Omega \gg t$, so the Floquet sectors decouple, we find that the effective Hamiltonian describing the $m=0$ sector is a $t$ - $J$ model with hopping $\tilde{t}=\mathcal{J}_{0}(v) t$, with $J$ and $\alpha$ unchanged. The ratio $J / \tilde{t}$ therefore becomes strongly driving dependent,

$$
J / \tilde{t}=\frac{4 t}{U \mathcal{J}_{0}(v)},
$$

and breaks the usual relation $J=4 t^{2} / U \ll t$. This allows the realization of the regime $J / \tilde{t}>1$ that is normally inaccessible.
Using this result, a slow increase in the driving strength $\dot{v} \ll J$ corresponds to adiabatically moving through the phase diagram of the $t-J$ model into this new parameter regime. For this reason, we now examine the equilibrium properties of this effective model and compare them to the driven steady states obtained in Sec. III.

\section{B. Phase diagram of the $t-J$ model}

In one dimension, the ground state of the $t-J$ model, for any $\alpha$ and below half-filling $\bar{n}<1$, is metallic with $K_{\rho}=0.5$ as $J / t \rightarrow 0$. As $J / t$ increases, $K_{\rho}$ also increases monotonically. When the hopping $t \ll J$, then $\hat{H}_{\text {ex }}+\hat{H}_{\text {pair }}$ together resemble a Hamiltonian for hard-core bosons. ${ }^{2}$ This similarity suggests that for sufficiently large $J / t$, superexchange mediates the formation of nearest-neighbor singlet pairs with binding energy $-J$, while the pair hopping gives them a bandwidth proportional to $\alpha J$. This is consistent with the form of the quasienergy spectrum near $v \approx v_{0}$ shown in Fig. 2(b).

The pairing effect is captured by simple energetic arguments. Solving the two-electron problem, the binding energy of nearest-neighbor singlet pairs is given by [53]

$$
E_{\text {pair }}=-J-2 \alpha J-\frac{4 t^{2}}{J+2 \alpha J} .
$$

Thus, in the dilute limit, comparing $E_{\text {pair }}$ to the energy for two free electrons $E_{\text {free }}=-4 t$ suggests that pair formation will occur for $J / t \geqslant 2 /(1+2 \alpha)$. These nearest-neighbor singlet pairs subsequently quasicondense to form a superconductor, characterized by dominant quasi-long-range singlet pair correlations [54],

$$
\left\langle\hat{b}_{j+x, j+1+x}^{\dagger} \hat{b}_{j, j+1}\right\rangle \sim x^{-\left(1+1 / K_{\rho}\right)},
$$

with $K_{\rho}>1$ signifying an attractive Luttinger liquid.

For yet larger $J / t$, the formation of phase-separated electron-rich and hole-rich regions signaled by a diverging $K_{\rho}$ might be expected [44]. The exact solution of a Heisenberg spin chain gives the antiferromagnetic bond energy $E_{\text {bonds }}=$ $-2 J \ln (2)$. For $\alpha=1 / 2$ we see that $E_{\text {pair }}<E_{\text {bonds }}$ for all values of $J / t$, meaning that singlet pairs will never freeze into larger antiferromagnetic clusters. The pair hopping term destabilizes antiferromagnetic clusters, even when $J / t \gg 1$, since it homogenizes holes throughout the system akin to hole repulsion. However, for $\bar{n} \geqslant 0.5$, superconductivity is not expected to persist up to $J / t \rightarrow \infty$. Instead, an RVB "singlet gas" appears with short-ranged pair correlations. This picture of the equilibrium properties of the one-dimensional (1D) $t-J$ model is borne out by comprehensive exact diagonalization [55], DMRG [44], and quantum Monte Carlo calculations [53].

The numerical results displayed in Figs. 3 and 4 are broadly consistent with these equilibrium properties of the $t-J$ model spanning a wide range of $J / t$ values. In particular, we may now attribute the increased peak height $P(q=0, \tau)$ and emergence of quasi-longer-ranged pair correlations to the driving elevating $J / t$ sufficiently slowly to near-adiabatically

\footnotetext{
${ }^{2}$ We note that while the $\hat{b}_{i j}$ and $\hat{b}_{i j}^{\dagger}$ operators commute on disjoint pairs of sites, they do not obey bosonic commutation relations if the pairs overlap.
} 

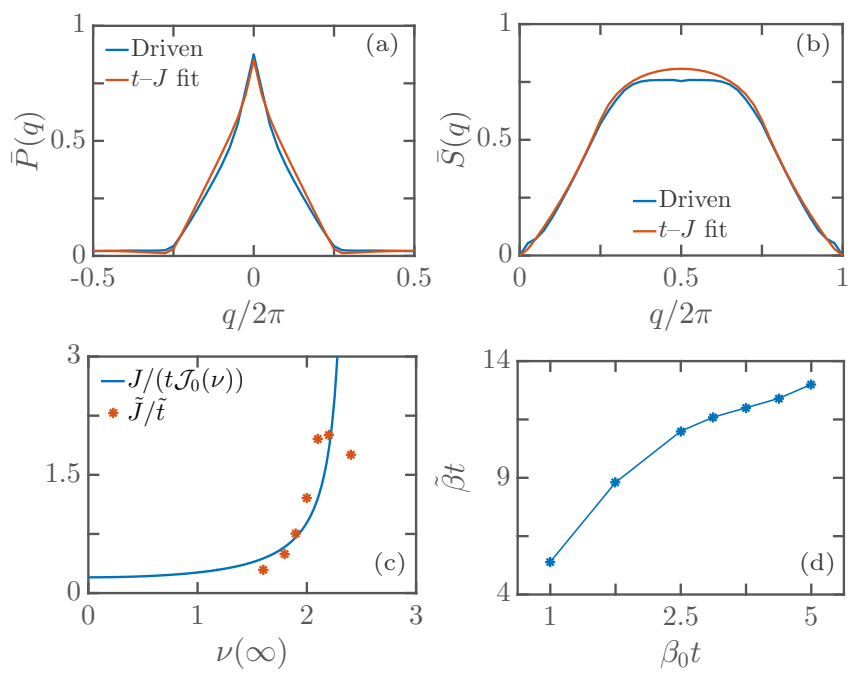

FIG. 6. The time average between $\tau t=60-80$ of the driven state's structure factors for (a) singlet pairing $\bar{P}(q)$ and (b) spin $\bar{S}(q)$ from Figs. 3(b) and 3(c) is shown alongside the same quantities for a $t$ - $J$ ground state with $\alpha=1 / 2$ and an effective $J_{\text {eff }} / t_{\text {eff }}$ that gives the closest match to $\bar{P}(q)$. For $v(\infty)=2.2$, the effective $J_{\text {eff }} / t_{\text {eff }}=2.0$. Repeating this fit for the sequence of driving strengths $v$ shown in Fig. 4(a) gives $J_{\text {eff }} / t_{\text {eff }}$ plotted in (c), with the solid curve reporting the prediction of Eq. (16). In (d) $\bar{P}(q)$ of the driven initial thermal state at inverse temperature $\beta_{0}$ was compared to $t$ - $J$ model thermal states, with $J$ fixed to its equilibrium value and the ratio $J / t$ fixed by the results in Fig. 6(c), to extract an effective inverse temperature $\beta_{\text {eff }}$. A solid line is drawn to guide the eye. The computations for (a)-(c) were carried out for $L=40$ sites, and those for (d) were carried out with $L=24$ sites.

transition the system from its initial metallic phase into the superconducting phase. This comparison is made more quantitatively in Figs. 6(a) and 6(b), where the singlet pairing and spin structure factors from Figs. 3(b) and 3(c) are closely matched to those of a $t-J$ model superconducting ground state with $J / t \approx 2$. For purposes of comparison with the $t$ - $J$ model, for simplicity we computed the driven Hubbard states and $t$ - $J$ ground states with the finite-size time-dependent DMRG algorithm with $L$ sites.

By repeating the fitting of the zero-temperature driven state singlet correlations to a $t-J$ model ground state, we extract an effective superexchange to hopping ratio $J_{\text {eff }} / t_{\text {eff }}$ as a function of driving strength $v$. The results in Fig. 6(c) show decent agreement with Eq. (16), and they indicate that the superconducting phase can be reached with $v(\infty) \approx 2$. We also fit the singlet structure factors of the driven state obtained from a finite initial temperature to thermal states of the $t-J$ model to obtain an effective temperature $\beta_{\text {eff }}$. The results, shown in Fig. 6(d), show that the driving substantially decreases the effective temperature, confirming our interpretation as adiabatically cooling the system.

\section{Floquet heating}

In general, periodic driving of a generic many-body system is expected to cause so-called Floquet heating, even far away from resonances. Finite $\Omega$ corrections will result in the
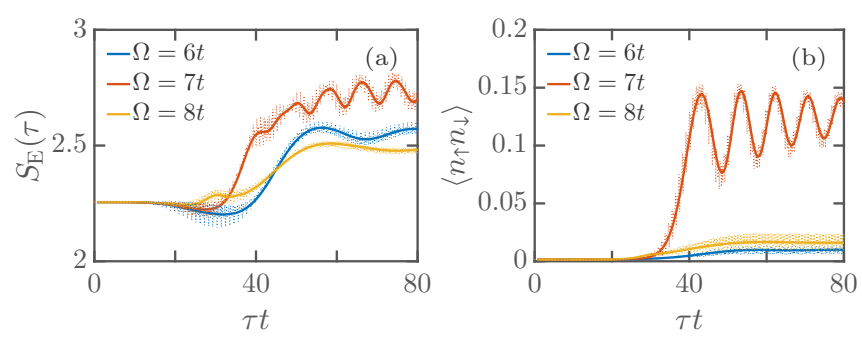

FIG. 7. (a) The entanglement entropy $S_{\mathrm{E}}$ between a two-site block and the rest of the zero-temperature, infinite system as a function of time. (b) The average double-occupancy per site $\left\langle n_{\uparrow} n_{\downarrow}\right\rangle$ is plotted. The dotted line shows the instantaneous value, while the solid line shows the moving time average. The system parameters not explicitly stated are the same as those in Fig. 3. Frequencies $\Omega=6 t$ and $7 t$ shown here correspond to the arrows drawn in Fig. 4(d).

system being described by an effective Hamiltonian possessing non-negligible "unphysical" terms that are both spatially nonlocal and multibody. Eigenstates of such a Hamiltonian will be highly delocalized in the eigenbasis of more physical short-ranged few-body Hamiltonians, such as the Hubbard and $t-J$ models. Thus the eigenstate thermalization hypothesis (ETH) [56-58] suggests that in the asymptotic long-time limit, independent of its initial state, any finite frequency drive results in all physical observables of the system becoming indistinguishable from those of a featureless infinite-temperature state [59-61]. However, the ETH does not predict the rate of Floquet heating [62].

Despite operating in a finite frequency driving regime, on the accessible simulation times the numerical results presented here do not display significant heating effects. The time-averaged correlations emerging from the driven Hubbard model, while possessing some small quantitative discrepancies, e.g., in Figs. 6(a) and 6(b), are nonetheless well captured by an effective $t-J$ model rather than a more pathological Hamiltonian. This suggests that the Floquet heating rate is small and that our findings are instead consistent with the notion of prethermalization in driven systems [63-66].

To examine the Floquet heating rate further, we compute the entanglement entropy between a two-site block and the rest of the infinite size, zero-temperature chain, as shown in Fig. 7(a). As expected, it increases when the driving is ramped up, showing that the driven state is more entangled compared to the initial Hubbard ground state. However, it quickly reaches a quasisteady state, and it does not show an appreciable further increase on the time scales considered, suggesting that Floquet heating is negligible here. The slow growth of entanglement in the system is precisely what allows our simulations to accurately track the dynamics for many 10's of hopping times. Even when the system is driven through the $\Omega=7 t$ resonance [shown in Fig. 4(d)], the entropy shows a negligible increase after the initial ramp. Although this resonant driving is rather energetic, creating and destroying doublons, it induces reversible dynamics [67], and thus it does not correspond to heating. Nonetheless, the creation of relatively large numbers of doublons highlights the breakdown of the effective $t-J$ model description in the resonant case, in 
contrast with the $\Omega=6 t$ drive, which avoids such resonances and maintains a small double occupancy.

The appearance of very slow Floquet heating rates for generic many-body systems was examined in several recent studies [68-71]. They show that an effective static Hamiltonian $\hat{H}_{*}$ will describe the system up to times $\tau<\tau_{*}$, where $\tau_{*} \propto \exp (C \Omega / \varepsilon)$. Here $C$ is a numerical constant of order unity, and $\varepsilon$ is an energy scale bounding the terms in the driven Hamiltonian [69]. Our results suggest that for in-gap driving, $\varepsilon \sim t$, giving a broad time window in which the system is described by an $\hat{H}_{*}$. This effective Hamiltonian may not be exactly a $t-J$ model, but it nonetheless appears to share its essential physical features, such as supporting a pairing phase. In such a broad time window, it is likely that dissipation and other couplings to the environment, which we do not model here, will govern the long time behavior. Floquet heating is therefore unlikely to be of dominant practical importance in experiments.

\section{CONCLUSIONS}

We have shown that periodic in-gap driving of a strongly correlated electronic system can slow electrons down while maintaining the normally subordinate superexchange interaction, making $J$ the dominant energy scale. This effect manifests itself in a many-body one-dimensional setting as a distinct switching of pair correlations. We showed that the driven state is similar to what would be expected if $J / t$ was enhanced to values that are considered unphysical in thermal equilibrium. Furthermore, these effects were found to be robust to finite ramp times of the driving and finite initial temperatures. Our future experimental and theory work is likely to focus on higher-dimensional systems in which dynamically enhanced pairing is expected to emerge at smaller values of $J / t$ [72]. The inclusion of competing instabilities, such as charge-density waves, and the interplay of driving with dissipation $[73,74]$ could then provide a fuller picture of this route to engineering light-induced superconducting states in quantum materials.

\section{ACKNOWLEDGMENTS}

This research is funded by the European Research Council under the European Union's Seventh Framework Programme (FP7/2007-2013)/ERC Grant Agreement No. 319286 QMAC. S.A. and D.J. acknowledge support from the EPSRC Tensor Network Theory grant (EP/K038311/1).

\section{APPENDIX A: FLOQUET HAMILTONIAN COUPLING}

In the main text, we concentrated on the special case of driving with $V_{a}=V_{b}=V$ and constant phase $\Delta \phi=\pi / 2$. Here we go back to the general driving $\hat{H}_{\text {drive }}$ given in Eq. (4) and define the Floquet-Fock basis as

$$
\begin{aligned}
\left|\left\{n_{j, \sigma}\right\}, m\right\rangle= & \left|\left\{n_{j, \sigma}\right\}\right\rangle \exp (i m \Omega \tau) \\
& \times \exp \left[-i \Delta_{a}(\tau) \sum_{j \in a} n_{j}-i \Delta_{b}(\tau) \sum_{j \in b} n_{j}\right],
\end{aligned}
$$

where $m$ is the Fourier component and

$$
\begin{aligned}
& \Delta_{a}(\tau)=-\frac{V_{a}}{2 \Omega} \cos (\Omega \tau-\Delta \phi), \\
& \Delta_{b}(\tau)=-\frac{V_{b}}{2 \Omega} \cos (\Omega \tau+\Delta \phi) .
\end{aligned}
$$

The Floquet Hamiltonian is defined by the eigenvalue problem Eq. (5), which in the basis Eq. (A1) becomes

$$
\left[\hat{H}(\tau)-i \partial_{\tau}\right]\left|\left\{n_{j, \sigma}\right\}, m\right\rangle=\left[\hat{H}_{\text {hop }}+\hat{H}_{\text {int }}+m \Omega \mathbb{1}\right]\left|\left\{n_{j, \sigma}\right\}, m\right\rangle \text {. }
$$

We then use the extended scalar product Eq. (6) to evaluate the right-hand side as $\left\langle\left\langle\left\{n_{j, \sigma}^{\prime}\right\}, m^{\prime}\left|\hat{H}_{\text {hop }}+\hat{H}_{\text {int }}+m \Omega \mathbb{1}\right|\left\{n_{j, \sigma}\right\}, m\right\rangle\right\rangle$. The last two terms are diagonal in the Floquet-Fock basis, so all the nontrivial physics is contained in the hopping matrix elements. These are given by

$$
\begin{aligned}
\left\langle\left\langle\left\{n_{j, \sigma}^{\prime}\right\}, m^{\prime}\left|\hat{H}_{\mathrm{hop}}\right|\left\{n_{j, \sigma}\right\}, m\right\rangle\right\rangle & \\
= & \frac{1}{T} \int_{0}^{T} \exp \left[i\left(m-m^{\prime}\right) \Omega \tau+i s \Delta_{a}(\tau)\right. \\
& \left.\quad-i s \Delta_{b}(\tau)\right] d \tau\left\langle\left\{n_{j, \sigma}^{\prime}\right\}\left|\hat{H}_{\mathrm{hop}}\right|\left\{n_{j, \sigma}\right\}\right\rangle, \\
= & \zeta_{m^{\prime}-m}\left\langle\left\{n_{j, \sigma}^{\prime}\right\}\left|\hat{H}_{\mathrm{hop}}\right|\left\{n_{j, \sigma}\right\}\right\rangle .
\end{aligned}
$$

Here we have used the fact that $\left\langle\left\{n_{j, \sigma}^{\prime}\right\}\left|\hat{H}_{\text {hop }}\right|\left\{n_{j, \sigma}\right\}\right\rangle$ is nonzero only when an electron (of either spin) moves from a site in sublattice $a$ to a site in sublattice $b$, or the reverse. Thus we can denote the change in the occupation of sublattice $a$ as $\sum_{j \in a}\left(n_{j}^{\prime}-n_{j}\right)=s$, and we know that the change in occupation of sublattice $b$ is $\sum_{j \in b}\left(n_{j}^{\prime}-n_{j}\right)=-s$, with $s= \pm 1$. The Floquet coupling coefficient $\zeta_{m^{\prime}-m}$ depends not only on the Fourier components but also on the parameters of the driving $V_{a}, V_{b}, \Delta \phi, \Omega$ and the Fock states being connected via $s$. To evaluate $\zeta_{m^{\prime}-m}$, we first expand $\Delta_{a}(\tau)-\Delta_{b}(\tau)$ as

$$
\begin{aligned}
\Delta_{a}(\tau)-\Delta_{b}(\tau)= & \frac{\left(V_{a}+V_{b}\right)}{2 \Omega} \sin (\Omega \tau) \sin (\Delta \phi) \\
& -\frac{\left(V_{a}-V_{b}\right)}{2 \Omega} \cos (\Omega \tau) \cos (\Delta \phi) .
\end{aligned}
$$

Next we use a Jacobi-Anger expansion to break up the exponentials of trigonometric functions and perform the timeaveraging integration to obtain

$$
\begin{aligned}
\zeta_{m^{\prime}-m}= & \sum_{n=-\infty}^{\infty}(-i)^{n} \mathcal{J}_{n}\left(s \frac{\left(V_{a}-V_{b}\right)}{2 \Omega} \cos (\Delta \phi)\right) \\
& \times \sum_{n^{\prime}=-\infty}^{\infty} \mathcal{J}_{n^{\prime}}\left(s \frac{\left(V_{a}+V_{b}\right)}{2 \Omega} \sin (\Delta \phi)\right) \delta_{n+n^{\prime}, m^{\prime}-m}
\end{aligned}
$$

This coupling appears in the Floquet Hamiltonian in Eq. (8) for the most general driving parameters. For the special case used in the main text, $V_{a}=V_{b}=V$ and $\Delta \phi=\pi / 2$, this reduces $\zeta_{m^{\prime}-m}$ in Eq. (A6) to that given in Eq. (9). However, note that Eq. (A6) displays suppression of the hopping for a much wider range of driving parameters than this special case. As an example, in Fig. 8 we take $v_{a}=V_{a} / \Omega=2 v_{b}$ and plot $\zeta_{0}$, the hopping suppression factor in the limit $U \rightarrow \infty, \Omega \rightarrow \infty$, and $U / \Omega<1$. This shows that the hopping can still be suppressed to zero even in the case in which $v_{a} \neq v_{b}$ and $\Delta \phi \neq \pi / 2$. 


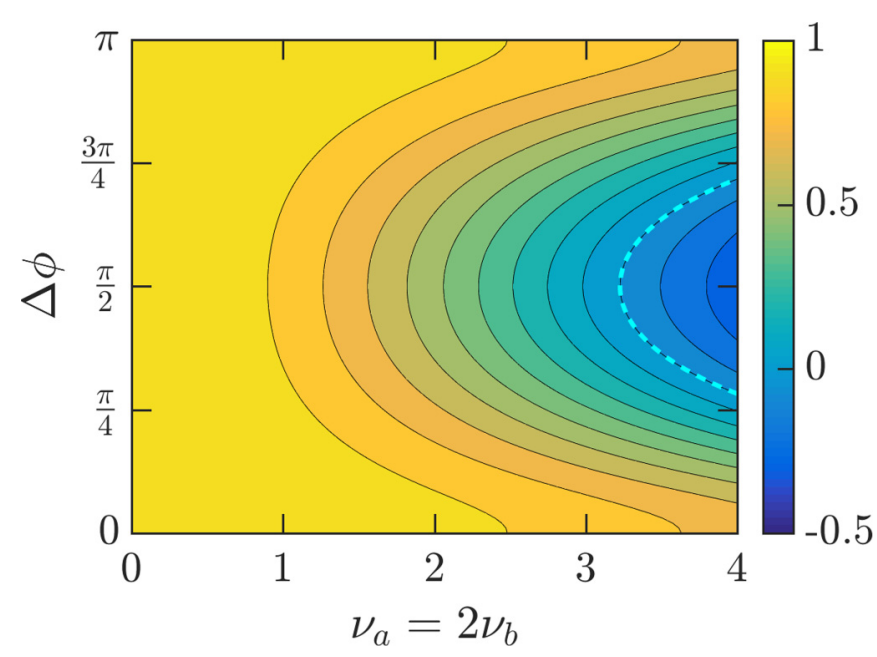

FIG. 8. The coefficient $\zeta_{0}$ with unequal $a$ and $b$ driving strengths as a function of $v_{a}$ and $\Delta \phi$. The dotted line marks the contour $\zeta_{0}=0$, where single-particle hopping is suppressed to 0 .

\section{APPENDIX B: CROSSINGS IN THE FLOQUET SPECTRUM}

As seen in Fig. 4(d), there are several resonance frequencies at which pairing (and indeed all correlations) is destroyed rather than enhanced by the driving. This can be understood by looking at the Floquet quasienergy spectrum. Since the spectrum is periodic, high-energy states in the upper Hubbard band are folded down into the first Brillouin zone $-\Omega / 2<$ $U-m \Omega<\Omega / 2$, as shown in Fig. 9 for a small system. For $\Omega=6 t$, folded upper Hubbard band states do not intersect the lower Hubbard band portion of the spectrum, and so a sufficiently slow ramp will adiabatically follow the Floquet state connected to the undriven ground state. However, for the $\Omega=7 t$ case, there is a level crossing between these

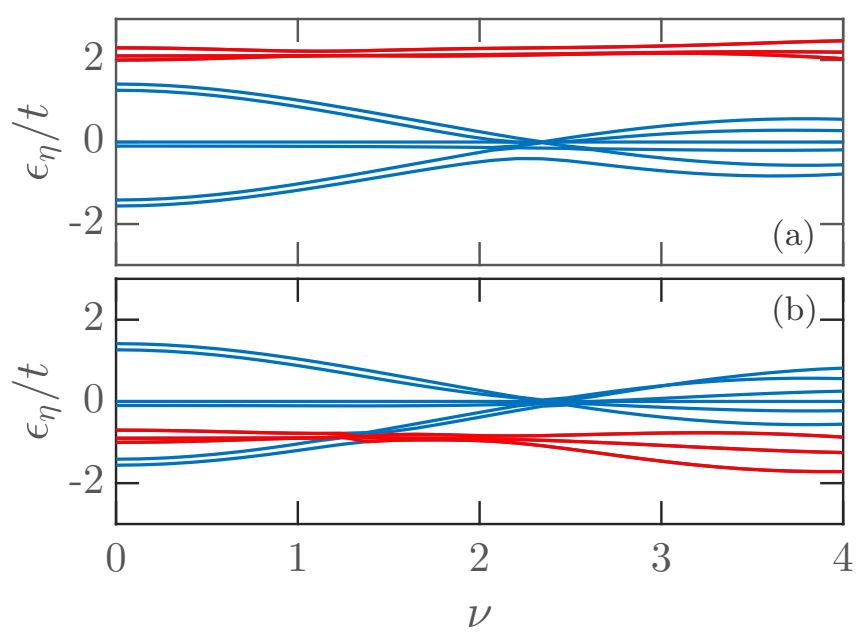

FIG. 9. The Floquet spectrum $\epsilon_{\eta}$ for an $L=6$ site system with $U=20 t, \Delta \phi=\pi$. The doubly occupied levels are drawn in red, the singly occupied are drawn in blue. In (a) $\Omega=6 t$, and there are no crossings between singly and doubly occupied states. In (b) $\Omega=7 t$, and we observe a crossing between the upper and lower Hubbard bands. The two frequencies shown here correspond to the arrows drawn in Fig. 4(d). bands. Similar crossings are found for the other resonance frequencies in Fig. 4(d). By driving through these crossings, strong excitation of states in the upper Hubbard band is expected, destroying nearest-neighbour singlet correlations. Indeed, for the larger system this is what is shown in Fig. 7(b), where driving at $\Omega=7 t$ is found to result in a 10 times larger increase in the average density of double occupancies than driving at $\Omega=6 t$. The smallness of the latter case supports our interpretation of the driven system with an effective $t-J$ model.

\section{APPENDIX C: DETAILS ON NUMERICAL CALCULATIONS}

For the zero-temperature calculations, we computed the ground state of the Hubbard system using the infinite timeevolving block decimation (iTEBD) to evolve an initial translationally invariant infinite matrix product state (iMPS) in imaginary time until a convergence in the singular values is reached [40]. Using a fourth-order Suzuki-Trotter decomposition, and successively refining the time step to systematically eliminate the Trotter error and bring the final state into canonical form, we obtain an iMPS with a bond dimension of $\chi=200$. We subsequently evolve this iMPS using iTEBD, and we compute the expectation values given in Sec. III A.

To accurately capture the driven state, our iTEBD calculations were performed with iMPS bond dimensions up to $\chi=400$ and a driving frequency-dependent time step $\Delta \tau=$ $2 \pi /(50 \Omega)$. To resolve the fast oscillations in the correlation functions, they were sampled every 10 time steps. The correlations were robust to moderate changes in $\chi$ and $\Delta \tau$, and the cumulative truncation error during the time evolution remained small.

In Fig. 10, we show the spin and singlet structure factors for the driven system for finite-size $(L=40)$ calculation, with the filling $n=1 / 2$, fixed using a U(1) symmetric MPS. We compare this to the results obtained for the infinite system using iTEBD results computed without symmetries, and a chemical potential $\mu=-0.2 t$ result. We find essentially no difference between the results for the finite and infinite systems, thus demonstrating that the results seen for the finite system are not a finite-size effect. Consequently, for simplicity and computational tractability, we use a finite system for the purposes of finite-temperature calculations and for comparisons to the $t-J$ model.
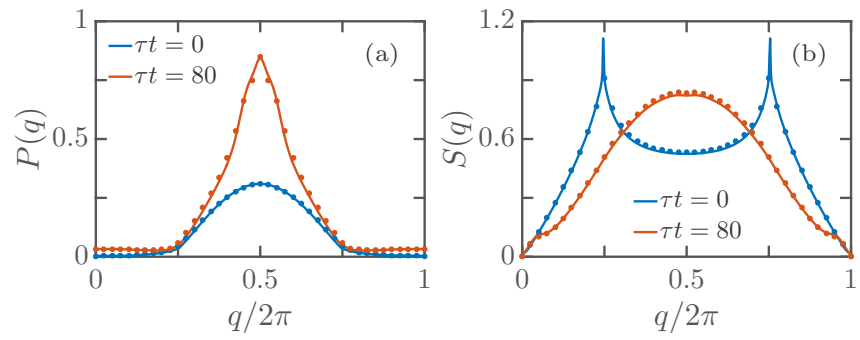

FIG. 10. A comparison of the (a) singlet and (b) spin structure factors at two different times, computed for the same parameters as in Fig. 3. The points show results for the finite-size, $L=40$ results, while the line shows the infinite-size results. 
To quantify the amount of Floquet heating in our system, we compute the entanglement entropy between a two-site block and the remainder of the infinite system, defined as

$$
S_{\mathrm{E}}(\tau)=-\operatorname{Tr}\left[\rho_{\mathrm{C}}(\tau) \log _{2}\left(\rho_{\mathrm{C}}(\tau)\right)\right],
$$

where $\rho_{\mathrm{C}}$ is the reduced density matrix for the two-site block. The behavior of $S_{\mathrm{E}}(\tau)$ is shown in Fig. 7(a) for various driving frequencies. After ramping up the driving, the entanglement entropy grows in time, quickly reaching a quasisteady state. Note that the maximum possible value of $S_{\mathrm{E}}$ (for an infinitetemperature state) is $\log _{2}(16)=4$. The largest growth is seen for $\Omega=7 t$, which was already identified in Fig. 4(d) as a Floquet resonance. The additional entanglement makes the resonantly driven case more difficult to capture with an MPS ansatz due to a rapid growth of truncation error after the ramp [75]. The growth in $S_{\mathrm{E}}(\tau)$ for frequencies away from resonances are broadly similar to each other and smaller. As we see no growth in $S_{\mathrm{E}}(\tau)$ after the ramp, we find no evidence of Floquet heating on the time scales considered here.

For the finite temperature calculations, we obtained thermal states of a finite-sized Hubbard model via imaginary-time TEBD. An initial matrix product operator (MPO) representation of the identity matrix (i.e., an infinite-temperature state) was evolved in imaginary time with a time step $\Delta \beta=0.01 / t$ to obtain an MPO representation of the desired thermal mixed state [48], tuning the chemical potential to obtain desired filling $\bar{n}=1 / 2$. We then performed real-time evolution with an MPO dimension up to $\chi=400$ to describe the driven state.

\section{APPENDIX D: DIFFERENT FILLINGS}

For clarity and its relevance to organic salts, in the main text we focused on quarter-filling. To confirm that drivingenhanced singlet pairing is still present away from half-filling, we repeated the iTEBD calculations presented in Fig. 3. However, we instead begin the time evolution from ground states obtained with chemical potentials $\mu=-1.0 t$ and $1.5 t$, resulting in fillings of $\bar{n} \approx 0.35$ and 0.82 , respectively. The results are shown in Fig. 11. We find that irrespective of the filling, we obtain qualitatively the same features as in Fig. 3. Namely, the sharp peaks in the spin structure factor $S(q)$ are suppressed in favor of a broad peak about $q=\pi$, and a significant enhancement in the singlet structure factor of about $q=0$ is again observed. However, as discussed in Sec. IVB, the one-dimensional $t$ - $J$ model with $\alpha=1 / 2$ features suppressed superconducting correlations at dense fillings, reflected here in the more modest pairing enhancement at $P(q=0)$.

\section{APPENDIX E: PERTURBATIVE CORRECTIONS}

The virtue of constructing a Floquet Hamiltonian $\hat{H}_{\mathrm{F}}$ is that standard time-independent perturbative methods are applicable to it. In particular, given a Hamiltonian $\hat{H}=\hat{H}_{0}+\lambda \hat{H}_{1}$, composed of a bare contribution $\hat{H}_{0}$ and a perturbation $\hat{H}_{1}$ with coupling strength $\lambda$, we wish to determine an effective Hamiltonian $\hat{H}_{\text {eff }}$ describing the energy eigenvalues of $\hat{H}$ in a degenerate subspace of eigenstates of $\hat{H}_{0}$ with energy $E$. To second order in $\lambda$ we obtain $\hat{H}_{\text {eff }}$ via a standard projection
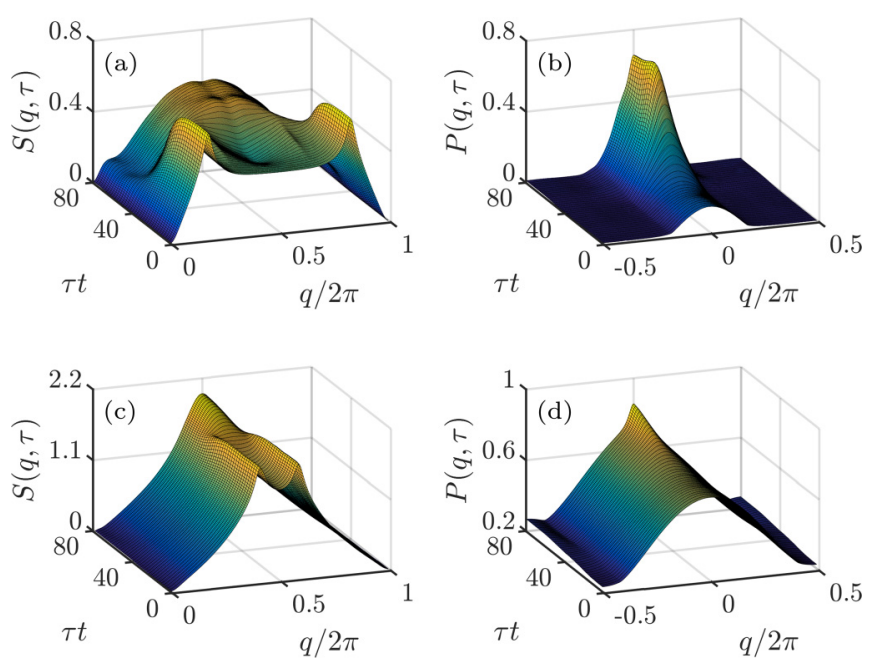

FIG. 11. An infinite Hubbard chain with the same system parameters as in Fig. 3, except for the chemical potential, which is $\mu=-1.0 t$ in (a) and (b), and $\mu=1.5 t$ in (c) and (d). The respective spin structure factors are shown in (a) and (c), while the singlet structure factors are shown in (b) and (d).

approach [52] as

$$
\hat{H}_{\mathrm{eff}}=\mathbb{P} \hat{H} \mathbb{P}-\mathbb{P} \hat{H} \mathbb{Q} \frac{1}{\mathbb{Q} \hat{H} \mathbb{Q}-E} \mathbb{Q} \hat{H} \mathbb{P},
$$

where $\mathbb{P}$ is the projector onto the degenerate subspace of $\hat{H}_{0}$, and $\mathbb{Q}$ is its orthogonal complement. For $\hat{H}=\hat{H}_{\text {hub }} / U$, where $\hat{H}_{0}=\hat{H}_{\text {int }} / U, \lambda \hat{H}_{1}=\hat{H}_{\text {hop }} / U$, and $\mathbb{P}=\mathbb{P}_{0}$, this method yields the $t-J$ model given in Eq. (12).

We now apply the same approach to $\hat{H}_{\mathrm{F}}$ in the enlarged Hilbert space $\mathcal{H} \otimes \mathcal{T}$ using a projector $\mathbb{P}=\mathbb{P}_{0} \mathbb{M}_{0}$, where $\mathbb{M}_{0}$ is the projector onto the $m=0$ Floquet sector, i.e., the dc Fourier component. This approach has the virtue of dealing with the strong-coupling limit and driving on the same footing [51]. Specifically, it allows us to determine an effective time-independent Hamiltonian describing the stroboscopic evolution that contains perturbative corrections due to couplings to doubly occupied states and their Floquet replicas. We find that this again yields the $t$ - $J$ model with $\alpha=1 / 2$, and $\tilde{t}=\mathcal{J}_{0}(v) t$ as before, but a superexchange coupling given by

$$
\tilde{J}=\tilde{J}_{\text {kin }}+\tilde{J}_{\text {light }},
$$

where

$$
\tilde{J}_{\text {kin }} / \tilde{t}=\frac{4 t \mathcal{J}_{0}(v)}{U}
$$

and

$$
\tilde{J}_{\text {light }} / \tilde{t}=\frac{4 t}{U \mathcal{J}_{0}(v)} \sum_{m=1}^{\infty}\left[\frac{\mathcal{J}_{m}(\nu)^{2}}{1+m \Omega / U}+\frac{\mathcal{J}_{m}(v)^{2}}{1-m \Omega / U}\right] .
$$

Note that this second-order result for the effective $\tilde{J}$ for sublattice driving is identical to that obtained by applying a driving term describing an ac electric field across the lattice [23]. In the limit $U \gg \Omega, v<1$, the expression for $\tilde{J}$ reduces to Eq. (16) found from the $t-J$ model Floquet analysis. 
In the limit of $\Omega \rightarrow \infty$, the energy separation between the Floquet sectors far exceeds the energy scales $t$ and $U$ within them. Consequently, the perturbative contributions to $\tilde{J}$ from $m \neq 0$ sectors can be safely ignored, leaving a suppressed superexchange given by $\tilde{J} / \tilde{t}=4 \tilde{t} / U$. Indeed, Eq. (E4) indicates that above-gap driving $\Omega>U$ will lead generically to a reduction in $\tilde{J}$. This is because while $m<0$ and $m>0$ contributions have the same numerator, for $m<$ 0 the denominator is negative and smaller than for $m>0$. It therefore acts against the $m \geqslant 0$ contributions to reduce $\tilde{J}$ pushing the system further into the metallic $\tilde{J} / \tilde{t} \ll 1$ regime.
For in-gap frequencies $t<\Omega<U$, an interesting interplay between strong interactions and driving leads to different behavior. In this case, the leading $m<0$ contributions, where $\mathcal{J}_{m}(v)^{2}$ is non-negligible, lie above the retained subspace, so their denominators are positive. These contributions represent superexchange processes in which the gap $U$ is bridged virtually by borrowing $m \Omega$ energy from the driving and then returned. The net effect of this is to strengthen the $m \geqslant 0$ contributions resulting in $\tilde{J}$ slightly increasing beyond its equilibrium value. Since the hopping continues to be suppressed, in-gap driving can therefore access the regime $\tilde{J} / \tilde{t}>1$.
[1] M. Rini, R. Tobey, N. Dean, J. Itatani, Y. Tomioka, Y. Tokura, R. W. Schoenlein, and A. Cavalleri, Control of the electronic phase of a manganite by mode-selective vibrational excitation, Nature (London) 449, 72 (2007).

[2] D. Fausti, R. I. Tobey, N. Dean, S. Kaiser, A. Dienst, M. C. Hoffmann, S. Pyon, T. Takayama, H. Takagi, and A. Cavalleri, Light-induced superconductivity in a stripe-ordered cuprate, Science 331, 189 (2011).

[3] F. Schmitt, P. S. Kirchmann, U. Bovensiepen, R. G. Moore, L. Rettig, M. Krenz, J.-H. Chu, N. Ru, L. Perfetti, D. H. Lu, M. Wolf, I. R. Fisher, and Z.-X. Shen, Transient electronic structure and melting of a charge density wave in $\mathrm{TbTe}_{3}$, Science $\mathbf{3 2 1}$, 1649 (2008).

[4] K. Miyano, T. Tanaka, Y. Tomioka, and Y. Tokura, Photoinduced Insulator-to-Metal Transition in a Perovskite Manganite, Phys. Rev. Lett. 78, 4257 (1997).

[5] A. Cavalleri, Cs. Tóth, C. W. Siders, J. A. Squier, F. Ráksi, P. Forget, and J. C. Kieffer, Femtosecond Structural Dynamics in $\mathrm{VO}_{2}$ During an Ultrafast Solid-Solid Phase Transition, Phys. Rev. Lett. 87, 237401 (2001).

[6] L. Perfetti, P. A. Loukakos, M. Lisowski, U. Bovensiepen, M. Wolf, H. Berger, S. Biermann, and A. Georges, Femtosecond dynamics of electronic states in the Mott insulator $1 \mathrm{~T}-\mathrm{TaS}_{2}$ by time resolved photoelectron spectroscopy, New J. Phys. 10, 053019 (2008).

[7] E. Beaurepaire, J.-C. Merle, A. Daunois, and J.-Y. Bigot, Ultrafast Spin Dynamics in Ferromagnetic Nickel, Phys. Rev. Lett. 76, 4250 (1996).

[8] C. D. Stanciu, F. Hansteen, A. V. Kimel, A. Kirilyuk, A. Tsukamoto, A. Itoh, and Th. Rasing, All-Optical Magnetic Recording with Circularly Polarized Light, Phys. Rev. Lett. 99, 047601 (2007).

[9] H. Ehrke, R. I. Tobey, S. Wall, S. A. Cavill, M. Först, V. Khanna, Th. Garl, N. Stojanovic, D. Prabhakaran, A. T. Boothroyd, M. Gensch, A. Mirone, P. Reutler, A. Revcolevschi, S. S. Dhesi, and A. Cavalleri, Photoinduced Melting of Antiferromagnetic Order in $\mathrm{La}_{0.5} \mathrm{Sr}_{1.5} \mathrm{MnO}_{4}$ Measured Using Ultrafast Resonant Soft X-Ray Diffraction, Phys. Rev. Lett. 106, 217401 (2011).

[10] M. Först, R. I. Tobey, S. Wall, H. Bromberger, V. Khanna, A. L. Cavalieri, Y.-D. Chuang, W. S. Lee, R. Moore, W. F. Schlotter, J. J. Turner, O. Krupin, M. Trigo, H. Zheng, J. F. Mitchell, S. S. Dhesi, J. P. Hill, and A. Cavalleri, Driving magnetic order in a manganite by ultrafast lattice excitation, Phys. Rev. B 84, 241104 (2011).
[11] M. Mitrano, A. Cantaluppi, D. Nicoletti, S. Kaiser, A. Perucchi, S. Lupi, P. Di Pietro, D. Pontiroli, M. Riccò, S. R. Clark, D. Jaksch, and A. Cavalleri, Possible light-induced superconductivity in $\mathrm{K}_{3} \mathrm{C}_{60}$ at high temperature, Nature (London) 530, 461 (2016).

[12] S. J. Denny, S. R. Clark, Y. Laplace, A. Cavalleri, and D. Jaksch, Proposed Parametric Cooling of Bilayer Cuprate Superconductors by Terahertz Excitation, Phys. Rev. Lett. 114, 137001 (2015).

[13] W. Hu, S. Kaiser, D. Nicoletti, C. R. Hunt, I. Gierz, M. C. Hoffmann, M. Le Tacon, T. Loew, B. Keimer, and A. Cavalleri, Optically enhanced coherent transport in $\mathrm{YBa}_{2} \mathrm{Cu}_{3} \mathrm{O}_{6.5}$ by ultrafast redistribution of interlayer coupling, Nat. Mater. 13, 705 (2014).

[14] S. Kaiser, C. R. Hunt, D. Nicoletti, W. Hu, I. Gierz, H. Y. Liu, M. Le Tacon, T. Loew, D. Haug, B. Keimer, and A. Cavalleri, Optically induced coherent transport far above $T_{c}$ in underdoped $\mathrm{YBa}_{2} \mathrm{Cu}_{3} \mathrm{O}_{6+\delta}$, Phys. Rev. B 89, 184516 (2014).

[15] R. Mankowsky, A. Subedi, M. Först, S. O. Mariager, M. Chollet, H. T. Lemke, J. S. Robinson, J. M. Glownia, M. P. Minitti, A. Frano, M. Fechner, N. A. Spaldin, T. Loew, B. Keimer, A. Georges, and A. Cavalleri, Nonlinear lattice dynamics as a basis for enhanced superconductivity in $\mathrm{YBa}_{2} \mathrm{Cu}_{3} \mathrm{O}_{6.5}$, Nature (London) 516, 71 (2014).

[16] M. A. Sentef, A. F. Kemper, A. Georges, and C. Kollath, Theory of light-enhanced phonon-mediated superconductivity, Phys. Rev. B 93, 144506 (2016).

[17] M. Knap, M. Badadi, G. Refael, I. Martin, and E. Demler, Dynamical Cooper pairing in non-equilibrium electron-phonon systems, Phys. Rev. B 94, 214504 (2016).

[18] A. Subedi, A. Cavalleri, and A. Georges, Theory of nonlinear phononics for coherent light control of solids, Phys. Rev. B 89, 220301 (2014).

[19] J. H. Shirley, Solution of the Schrödinger equation with a Hamiltonian periodic in time, Phys. Rev. 138, B979 (1965).

[20] D. H. Dunlap and V. M. Kenkre, Dynamic localization of a charged particle moving under the influence of an electric field, Phys. Rev. B 34, 3625 (1986).

[21] A. Buchleitner, D. Delande, and J. Zakrzewski, Non-dispersive wave packets in periodically driven quantum systems, Phys. Rep. 368, 409 (2002).

[22] M. Bukov, L. D'Alessio, and A. Polkovnikov, Universal high-frequency behavior of periodically driven systems: From dynamical stabilization to Floquet engineering, Adv. Phys. 64, 139 (2015). 
[23] J. Mentink, K. Balzer, and M. Eckstein, Ultrafast and reversible control of the exchange interaction in Mott insulators, Nat. Commun. 6, 6708 (2015).

[24] C. E. Creffield and G. Platero, AC-driven localization in a twoelectron quantum dot molecule, Phys. Rev. B 65, 113304 (2002).

[25] A. Eckardt, C. Weiss, and M. Holthaus, Superfluid-Insulator Transition in a Periodically Driven Optical Lattice, Phys. Rev. Lett. 95, 260404 (2005).

[26] S. Kaiser, S. R. Clark, D. Nicoletti, G. Cotugno, R. I. Tobey, N. Dean, S. Lupi, H. Okamoto, T. Hasegawa, D. Jaksch, and A. Cavalleri, Optical properties of a vibrationally modulated solid state Mott insulator, Sci. Rep. 4, 3823 (2014).

[27] R. Singla, G. Cotugno, S. Kaiser, M. Först, M. Mitrano, H. Y. Liu, A. Cartella, C. Manzoni, H. Okamoto, T. Hasegawa, S. R. Clark, D. Jaksch, and A. Cavalleri, THz-Frequency Modulation of the Hubbard $U$ in an Organic Mott Insulator, Phys. Rev. Lett. 115, 187401 (2015).

[28] H. Mori, S. Tanaka, and T. Mori, Systematic study of the electronic state in $\theta$-type BEDT-TTF organic conductors by changing the electronic correlation, Phys. Rev. B 57, 12023 (1998).

[29] A. Eckardt, M. Holthaus, H. Lignier, A. Zenesini, D. Ciampini, O. Morsch, and E. Arimondo, Exploring dynamic localization with a Bose-Einstein condensate, Phys. Rev. A 79, 013611 (2009).

[30] G. Jotzu, M. Messer, R. Desbuquois, M. Lebrat, T. Uehlinger, D. Greif, and T. Esslinger, Experimental realization of the topological Haldane model with ultracold fermions, Nature (London) 515, 237 (2014)

[31] I. Bloch, J. Dalibard, and W. Zwerger, Many-body physics with ultracold gases, Rev. Mod. Phys. 80, 885 (2008).

[32] M. Lewenstein, A. Sanpera, and V. Ahufinger, Ultracold Atoms in Optical Lattices: Simulating Quantum Many-body Systems (Oxford University Press, Oxford, 2012).

[33] J. Struck, C. Ölschläger, M. Weinberg, P. Hauke, J. Simonet, A. Eckardt, M. Lewenstein, K. Sengstock, and P. Windpassinger, Tunable Gauge Potential for Neutral and Spinless Particles in Driven Optical Lattices, Phys. Rev. Lett. 108, 225304 (2012).

[34] R. Desbuquois, M. Messer, F. Görg, K. Sandholzer, G. Jotzu, and T. Esslinger, Controlling the Floquet state population and observing micromotion in a periodically driven two-body quantum system, arXiv:1703.07767.

[35] T. Bilitewski and N. R. Cooper, Synthetic dimensions in the strong-coupling limit: Supersolids and pair superfluids, Phys. Rev. A 94, 023630 (2016).

[36] H. Lignier, C. Sias, D. Ciampini, Y. Singh, A. Zenesini, O. Morsch, and E. Arimondo, Dynamical Control of Matter-Wave Tunneling in Periodic Potentials, Phys. Rev. Lett. 99, 220403 (2007).

[37] U. Schollwöck, The density-matrix renormalization group in the age of matrix product states, Ann. Phys. (N.Y.) 326, 96 (2011).

[38] F. Verstraete, V. Murg, and J. I. Cirac, Matrix product states, projected entangled pair states, and variational renormalization group methods for quantum spin systems, Adv. Phys. 57, 143 (2008).

[39] G. Vidal, Efficient Classical Simulation of Slightly Entangled Quantum Computations, Phys. Rev. Lett. 91, 147902 (2003).

[40] G. Vidal, Classical Simulation of Infinite-Size Quantum Lattice Systems in One Spatial Dimension, Phys. Rev. Lett. 98, 070201 (2007).
[41] S. Al-Assam, S. R. Clark, and D. Jaksch, Tensor network theory-Part 1: Overview of core library and tensor operations, arXiv: 1610.02244.

[42] T. Giamarchi, Quantum Physics in One Dimension (Oxford University Press, Oxford, 2004).

[43] S. Ejima, F. Gebhard, and S. Nishimoto, Tomonaga-Luttinger parameters for doped Mott insulators, Europhys. Lett. 70, 492 (2005).

[44] A. Moreno, A. Muramatsu, and S. R. Manmana, Ground-state phase diagram of the one-dimensional $t$ - $J$ model, Phys. Rev. B 83, 205113 (2011).

[45] Y. C. Chen and T. K. Lee, New phase in the one-dimensional $t$ - $J$ model, Phys. Rev. B 47, 11548 (1993).

[46] A. M. Läuchli and C. Kollath, Spreading of correlations and entanglement after a quench in the one-dimensional BoseHubbard model, J. Stat. Mech. (2008) P05018.

[47] F. Verstraete, J. J. García-Ripoll, and J. I. Cirac, Matrix Product Density Operators: Simulation of Finite-Temperature and Dissipative Systems, Phys. Rev. Lett. 93, 207204 (2004).

[48] M. Zwolak and G. Vidal, Mixed-State Dynamics in OneDimensional Quantum Lattice Systems: A Time-Dependent Superoperator Renormalization Algorithm, Phys. Rev. Lett. 93, 207205 (2004).

[49] P. B. Blakie and A. Bezett, Adiabatic cooling of fermions in an optical lattice, Phys. Rev. A 71, 033616 (2005).

[50] S. Sotiriadis, P. Calabrese, and J. Cardy, Quantum quench from a thermal initial state, Europhys. Lett. 87, 20002 (2009).

[51] M. Bukov, M. Kolodrubetz, and A. Polkovnikov, SchriefferWolff Transformation for Periodically Driven Systems: Strongly Correlated Systems with Artificial Gauge Fields, Phys. Rev. Lett. 116, 125301 (2016).

[52] F. H. L. Essler, H. Frahm, F. Gohmann, A. Klumper, and V. E. Korepin, The One-dimensional Hubbard Model (Cambridge University Press, Cambridge, 2005).

[53] B. Ammon, M. Troyer, and H. Tsunetsugu, Effect of the three-site hopping term on the $t-J$ model, Phys. Rev. B 52, 629 (1995).

[54] J. Spałek, Effect of pair hopping and magnitude of intra-atomic interaction on exchange-mediated superconductivity, Phys. Rev. B 37, 533 (1988).

[55] M. Ogata, M. U. Luchini, S. Sorella, and F. F. Assaad, Phase Diagram of the One-Dimensional $t$ - $J$ Model, Phys. Rev. Lett. 66, 2388 (1991).

[56] J. M. Deutsch, Quantum statistical mechanics in a closed system, Phys. Rev. A 43, 2046 (1991).

[57] M. Srednicki, Chaos and quantum thermalization, Phys. Rev. E 50, 888 (1994).

[58] M. Rigol, V. Dunjko, and M. Olshanii, Thermalization and its mechanism for generic isolated quantum systems, Nature (London) 452, 854 (2008).

[59] A. Lazarides, A. Das, and R. Moessner, Equilibrium states of generic quantum systems subject to periodic driving, Phys. Rev. E 90, 012110 (2014).

[60] L. D'Alessio and M. Rigol, Long-time behavior of isolated periodically driven interacting lattice systems, Phys. Rev. X 4, 041048 (2014).

[61] P. Ponte, A. Chandran, Z. Papić, and D. A. Abanin, Periodically driven ergodic and many-body localized quantum systems, Ann. Phys. (N.Y.) 353, 196 (2015). 
[62] M. M. Maricq, Application of average Hamiltonian theory to the NMR of solids, Phys. Rev. B 25, 6622 (1982).

[63] J. Berges, Sz. Borsányi, and C. Wetterich, Prethermalization, Phys. Rev. Lett. 93, 142002 (2004).

[64] M. Eckstein, M. Kollar, and P. Werner, Thermalization After an Interaction Quench in the Hubbard Model, Phys. Rev. Lett. 103, 056403 (2009).

[65] L. Mathey and A. Polkovnikov, Light cone dynamics and reverse Kibble-Zurek mechanism in two-dimensional superfluids following a quantum quench, Phys. Rev. A 81, 033605 (2010).

[66] M. Bukov, M. Heyl, D. A. Huse, and A. Polkovnikov, Heating and many-body resonances in a periodically driven two-band system, Phys. Rev. B 93, 155132 (2016).

[67] J. J. Mendoza-Arenas, F. J. Gomez-Ruiz, M. Eckstein, D. Jaksch, and S. R. Clark, Ultra-fast control of magnetic relaxation in a periodically driven Hubbard model, Ann. Phys. (Berlin) 2017, 1700024.

[68] D. A. Abanin, W. De Roeck, and F. Huveneers, Exponentially Slow Heating in Periodically Driven Many-Body Systems, Phys. Rev. Lett. 115, 256803 (2015).
[69] D. A. Abanin, W. De Roeck, and W. W. Ho, Effective Hamiltonians, prethermalization and slow energy absorption in periodically driven many-body systems, Phys. Rev. B 95 , 014112 (2017).

[70] T. Kuwahara, T. Mori, and K. Saito, Floquet-Magnus theory and generic transient dynamics in periodically driven many-body quantum systems, Ann. Phys. (N.Y.) 367, 96 (2016).

[71] T. Mori, T. Kuwahara, and K. Saito, Rigorous Bound on Energy Absorption and Generic Relaxation in Periodically Driven Quantum Systems, Phys. Rev. Lett. 116, 120401 (2016).

[72] S. Sorella, G. B. Martins, F. Becca, C. Gazza, L. Capriotti, A. Parola, and E. Dagotto, Superconductivity in the TwoDimensional $t$ - $J$ Model, Phys. Rev. Lett. 88, 117002 (2002).

[73] M. Langemeyer and M. Holthaus, Energy flow in periodic thermodynamics, Phys. Rev. E 89, 012101 (2014).

[74] H. Dehghani, T. Oka, and A. Mitra, Dissipative Floquet topological systems, Phys. Rev. B 90, 195429 (2014).

[75] A. J. Daley, S. R. Clark, D. Jaksch, and P. Zoller, Numerical analysis of coherent many-body currents in a single atom transistor, Phys. Rev. A 72, 043618 (2005). 